\title{
Does patient knowledge affect the colorectal cancer risk in ulcerative colitis?
}

\author{
J A Eaden, K Abrams, J F Mayberry
}

Postgrad Med J 2002;78:615-618

See end of article for authors' affiliations

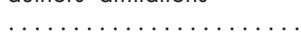

Correspondence to: Dr Jayne Eaden,

Department of

Gastroenterology,

Walsgrave Hospital,

Clifford Bridge Road,

Coventry CV2 2DX, UK;

jayne.eaden@btinternet.com

Submitted 25 April 2002

Accepted 22 July 2002

\begin{abstract}
Objectives: Formal efforts to improve patient education are associated with fewer disease complications in a number of conditions. The possible relationship between knowledge about ulcerative colitis and its cancer risk, and the development of colorectal cancer using a previously developed and validated instrument-the Crohn's and colitis knowledge (CCKNOW) score-were investigated.

Methods: The 24 item CCKNOW questionnaire was mailed to patients known to have developed colorectal cancer as a complication of ulcerative colitis (cases) and to colitics from the Leicestershire inflammatory bowel disease patient database who had not developed cancer (controls).

Results: The mean (SD) CCKNOW scores for cases was 8.21 (3.02) and for controls was 8.27 (4.3). These scores did not differ significantly between cases and controls (difference $0.06,95 \%$ confidence interval $(\mathrm{Cl})-1.7$ to $1.5, \mathrm{p}=0.9)$. There were four times as many members of the National Association of Crohn's and Colitis (NACC) in the control group compared with the cancer group and patients who are members of NACC achieve statistically significantly higher scores than non-members $(11.6 \vee 7.8$, $\mathrm{p}=0.05,95 \% \mathrm{Cl}-0.1$ to 7.6 ). However, after adjusting for NACC membership, the CCKNOW score did not appear to be associated with having developed cancer lodds ratio $1.04,95 \% \mathrm{Cl} 0.92$ to 1.18 , $\mathrm{p}=0.5$ ).

Conclusions: The CCKNOW scores were comparable in cases and controls. Thus, in a retrospective study, no evidence has been demonstrated of an association between patient knowledge and the risk of developing colorectal cancer in patients with ulcerative colitis. However, knowledge may have been increased in cases as a direct result of having had colorectal cancer as a complication of ulcerative colitis.
\end{abstract}

$\mathrm{T}$ he effectiveness of patient education on improving patient self management has been well documented in a variety of diseases. Increasing knowledge in patients with type 1 diabetes mellitus improves compliance with medication and glycated haemoglobin levels. ${ }^{12}$ A beneficial effect has also been demonstrated in asthmatics. Patients who underwent an education programme and consequently improved their asthma knowledge had fewer hospitalisations, fewer visits to family physicians, and reduced attendances at accident and emergency departments. ${ }^{3}$ Patient education has also been shown to be key in the outpatient management of thromboembolic disease. ${ }^{4}$ A well organised, structured education programme enabled patients to learn the necessary skills that permit complex and valuable therapies to be managed on an outpatient basis.

As improving knowledge in chronic disorders such as diabetes and asthma appears to reduce the frequency of complications, we postulated that good knowledge levels may be associated with fewer complications in patients with ulcerative colitis. We feel that patients who understand colitis and its cancer risk are more likely to manage their disease appropriately by attending surveillance colonoscopies and complying with medication. In this way such patients may have a lower mortality from colorectal cancer as a complication of ulcerative colitis through earlier detection. There will never be definite proof that colonoscopic surveillance influences overall mortality as there have never been any randomised controlled trials of surveillance in ulcerative colitis. Indeed it would now be unethical to conduct such a study. However, there is some evidence that the five year survival rate of those who participate in colonoscopic surveillance is improved..$^{5-7}$ In addition, although surveillance may be imperfect, it is currently recom- mended as the best method of detection of dysplasia or cancer at a surgically curable stage. ${ }^{8}$

There is growing interest in patient education in inflammatory bowel disease (IBD) and recently a new questionnaire, with good internal consistency and validity, has been developed to measure such knowledge. ${ }^{9}$ This instrument, the Crohn's and colitis knowledge (CCKNOW) score, encompasses four main areas and gives a total score representing overall knowledge about IBD. The purpose of the present study was to examine the relationship between knowledge about IBD and the development of colorectal cancer using the CCKNOW score.

\section{METHOD}

The development of the tool used to evaluate knowledge, the CCKNOW score, has previously been described in an earlier paper. ${ }^{9}$ It is a 24 item multiple choice questionnaire that assesses knowledge in the following areas of IBD management: (a) general IBD understanding, (b) medication, (c) diet, and (d) complications of IBD. The CCKNOW score is self administered with a scoring system of one point for each correct answer, and no negative marking. It has been shown to be a reliable index with high levels of internal consistency and a good level of readability.

Cases of colorectal cancer complicating ulcerative colitis nationwide were identified from a previous study conducted by the authors. ${ }^{10}$ Hospital records were checked to ensure that

Abbreviations: CCKNOW score, Crohn's and colitis knowledge score; $\mathrm{Cl}$, confidence interval; IBD, inflammatory bowel disease; NACC, National Association of Crohn's and Colitis 
Table 1 Characteristics of cases and controls

\begin{tabular}{lll}
\hline & Cases $(n=42)$ & Controls $(n=44)$ \\
\hline Gender (\%) & $28(67)$ & $31(70)$ \\
Male & $14(33)$ & $13(30)$ \\
Female & $59.9(12.1)$ & $59.8(10.2)$ \\
Mean (SD) age in years & $12.9(2.9)$ & $12.4(3.9)$ \\
Mean (SD) years spent in full time education & $2(5)$ & $8(18)$ \\
NACC membership (\%) & $8.2(3.0)$ & $8.3(4.3)$ \\
Mean (SD) score on 24 item CCKNOW & $5(12)$ & $8(18)$ \\
Number (\%) with good score $(>11)$ on the CCKNOW & & \\
\hline
\end{tabular}

cases were alive and approval of consultants in charge of patient care was sought before mailing the CCKNOW score to them. Seventy questionnaires were posted to cases. One hundred controls (matched for age and sex) identified from the Leicestershire IBD patient database were also mailed a CCKNOW score. These controls were not the same patients as those used in developing the CCKNOW score described in the previous paper. ${ }^{9}$

The level of statistical significance between the mean scores of cases and controls was estimated using an independent two sample $t$ test and 95\% confidence intervals were calculated. ${ }^{11}$ Assessment of the statistical significance of an association between being a case and a variety of potentially important characteristics was undertaken using logistic regression in SPSS. The study has a power of $70 \%$ to detect an odds ratio of 3.0 at the $5 \%$ significance level assuming an average exposure in the control group of $30 \%$.

\section{RESULTS}

After one mailing 42 questionnaires were returned by the cases, who had colorectal cancer complicating ulcerative colitis (response rate $60 \%$ ) and 44 were returned from the controls, who had ulcerative colitis (response rate $44 \%$ ). The characteristics of cases and controls are shown in table 1. There was no significant difference in the two groups in terms of age, gender, and the length of time spent in full time education. However, there were four times as many members of the National Association of Crohn's and Colitis (NACC) in the control group compared with the cases.

The mean score of the patients who had developed colorectal cancer complicating ulcerative colitis was 8.21 with a SD in their score of 3.02 (range 0-13). Controls had similar scores with a mean of 8.27 (SD 4.3, range 0-22). An independent two sample $t$ test showed that there was no statistically significant difference between the mean scores of the two groups (difference $0.06,95 \%$ confidence interval (CI) -1.7 to $1.5, \mathrm{p}=0.9$ ).

Scores were analysed according to membership of the NACC. The mean (SD) score for patients who were members, irrespective of being a case or a control, was 11.6 (5.3) whereas the mean scores for non-members was 7.8 (3.2). This difference was statistically significant (difference $3.8,95 \%$ CI -0.1 to $7.6, p=0.05$ ). We investigated the possibility of NACC membership having a bias and so the analysis was repeated after excluding the self help groups members. This reduced the mean scores in both groups but to a greater extent in the control group. The new mean scores were 8.13 for cases and 7.44 for controls respectively-that is, patients who had had colorectal cancer now achieved higher knowledge scores than those who had not. However, this difference is still not statistically significant (difference $0.69,95 \% \mathrm{CI}-0.8$ to $2.2, \mathrm{p}=0.4$ ). Therefore, NACC members were included in the rest of the analyses.

Logistic regression analysis found no statistically significant associations between scores on the CCKNOW, gender, age, and years spent in full time education. Being a member of NACC
Table 2 CCKNOW scores and cancer stage at diagnosis among cases

\begin{tabular}{lllll}
\hline & \multicolumn{4}{l}{ Score on CCKNOW questionnaire } \\
\cline { 2 - 5 } Dukes's stage & $<6$ & $7-12$ & $13-18$ & $19-24$ \\
\hline A & 2 & 10 & 0 & 0 \\
B & 2 & 12 & 0 & 0 \\
C & 3 & 11 & 1 & 0 \\
D & 0 & 1 & 0 & 0 \\
\hline
\end{tabular}

was associated with a reduction in the risk of developing colorectal cancer by $78 \%$ and scoring more than 11 on the CCKNOW reduced risk by $39 \%$ but these findings did not reach statistical significance $(p=0.07$ and $p=0.42$ respectively) possibly due to the relatively small numbers. After adjusting for NACC membership, the CCKNOW score did not appear to be associated with having developed cancer (odds ratio $1.04,95 \%$ CI 0.92 to $1.18, \mathrm{p}=0.5$ ).

To determine whether those cases who were diagnosed at a later Dukes's stage of colon cancer were those who had poorer knowledge levels, the CCKNOW scores were stratified according to the Dukes's stage of cancer at diagnosis among the cases who responded to the questionnaire (table 2 ). The data shows no correlation between the CCKNOW score and cancer stage at diagnosis.

The response rate among cases and controls was $60 \%$ and $44 \%$ respectively. The CCKNOW questionnaire was mailed only once. Hospital records were used to identify whether cases were deceased. These were not as accurate as was hoped and in two instances the questionnaire was returned by a relative stating that the patient had died. Concern was raised that this may have been the reason why other questionnaires among cases had not been returned. In order to avoid any possible anxiety to relatives the non-responders were not sent any further questionnaires. Consequently, so that both groups were treated uniformly, the CCKNOW questionnaire was not posted to non-responding controls.

The medical records of the cases had already been reviewed in a previous study. ${ }^{10}$ These data was reviewed to investigate whether there were any differences in the responding and non-responding cases. There were no statistically significant differences between responders and non-responders in terms of gender, extent of disease, and stage of cancer when diagnosed.

Considering questions from the general knowledge section of the questionnaire individually, both cases and controls (74\% and $68 \%$ respectively) knew that just because they may have been symptom-free for three years they were not cured of their condition but only a fifth (21\% and $23 \%)$ knew that IBD runs in families. A similar number in each group (only 14\% and 16\%) realised that a child with IBD may not grow to be as tall as his or her friends, with the remaining responders actually believing that they may be either less intelligent or may not live beyond 
the age of 45. A difference between the two groups was noted in the question concerning whether IBD can affect parts of the body other than the bowel. Only $26 \%$ of cases answered this correctly compared with $46 \%$ of the controls.

Regarding medication there was some confusion between the types of drug used to treat IBD. A similar number (57\% and $55 \%$ ) understood the role of immunosuppressive drugs but more controls knew that azathioprine was an example of such a drug ( $18 \% v 5 \%)$. Many more controls ( $73 \%$ v 55\%) knew that sulphasalazine was used to reduce the frequency of relapse and were aware that it can reversibly reduce male fertility $(27 \% v$ $5 \%)$. Concerning steroids an equivalent number $(50 \%$ of cases and $55 \%$ of controls) knew that they can be administered rectally and intravenously as well as orally.

As far as complications of IBD are concerned, perhaps not surprisingly more cases (29\% $v 18 \%)$ were aware which patients were at increased risk of bowel cancer and therefore who should be under surveillance. However, even with a personal history of cancer complicating colitis this is still a surprisingly small number. Fourteen per cent of cases thought that if they passed blood in their stool it meant that they definitely had bowel cancer compared with $9 \%$ of controls. Again more cases understood what a fistula was (43\% v 25\%) and had a better knowledge of bowel anatomy with $41 \%$ knowing the location of the terminal ileum compared to $30 \%$ of controls.

\section{DISCUSSION}

The level of knowledge in patients with ulcerative colitis is the same irrespective of whether they had developed colorectal cancer or not. Thus patient knowledge does not appear to affect the risk in patients with ulcerative colitis. We expected that cases would have significantly lower knowledge levels than controls as we anticipated that poor knowledge could be a risk factor. We based this on the assumption that patients with poor knowledge would be less likely to attend surveillance colonoscopies and comply with medication. Both of these factors have been shown to have a protective effect against cancer in colitis..$^{12} 13$

However, we feel that our study could be biased. There is a strong possibility that the knowledge level of cases has been significantly modified by the process of having colorectal cancer. It is likely that cases would have learnt more about their condition as a direct result of having cancer and the need to undergo surgery. Our data would support this view in that cases had notably better scores on anatomy questions and more knew who was at risk of bowel cancer compared with the control group. Controls achieved the same or better scores in other sections, knowing more about 5 -aminosalicylic acid medications and IBD affecting other parts of the body. It may be of significance that controls were more knowledgeable about sulphasalazine and mesalazine as these medications may be protective against colorectal cancer in ulcerative colitis. $^{101213}$

It was not possible to investigate differences between the cancer survivors who responded to the study and patients who had died from cancer complicating colitis. However, it is worth considering the possibility that patients who died may be those who were diagnosed at a later stage and these may also be the cases who were less knowledgeable. The study could be improved by obtaining further data from the medical records of the cases and controls such as disease duration and severity of disease as this could ostensibly affect the level of knowledge. Data such as missed colonoscopy and outpatient clinic appointments could be extracted and a comparison made between responding and non-responding cases. This would investigate the hypothesis that non-responders were those who were less compliant. However, obviously no data could be obtained concerning non-responding patients' knowledge levels.
Our findings are based on a small sample size and this limits any interpretation of the results. However, it is interesting to note that there were more members of the NACC in the control group. NACC members achieved significantly higher scores on the questionnaire which agrees with the findings in our previous study. 'We have shown a positive but nonsignificant association between membership of NACC and a reduction in the risk of developing colonic cancer. If this hypothesis is correct, could that protection be mediated through better knowledge or is it simply because they are a highly motivated group who attend surveillance and comply with medication? This is an interesting concept that merits further investigation through the continued use of the CCKNOW score in clinical practice.

The data showed no correlation between the CCKNOW score and the stage of colon cancer at diagnosis. However, this study was conducted retrospectively. Thus the current level of knowledge does not necessarily reflect the level of knowledge when the cancer was diagnosed. Ideally this study should be conducted prospectively with all patients completing the CCKNOW score at regular intervals, such as outpatient consultations. The CCKNOW could be used in its 12 item form (giving patients the two different versions alternately and periodically adding new questions) to prevent patients from learning answers. In this way we could obtain a true indication of the knowledge level of cases before developing cancer and be able to assess whether poor knowledge is a significant risk factor. However, such an approach would take at least 10 years and possibly as long as 30 to complete as the vast majority of colorectal cancers in ulcerative colitis occur after the first decade of disease. ${ }^{14-16}$ Easier outcomes to assess would be frequency of flare ups or the number of missed outpatient and colonoscopy appointments. These measures of compliance could be surrogate markers for the likelihood of developing cancer but this has not yet been assessed. This concept could easily be investigated in the future to determine whether knowledge can be used as a measure of such behaviours as adherence to therapeutic regimens or medical outcomes including reduced frequency of complications.

\section{Authors' affiliations}

J A Eaden, Department of Gastroenterology, Walsgrave Hospital, Coventry

K Abrams, Department of Epidemiology and Public Health, University of Leicester, Leicester

J F Mayberry, Gastrointestinal Research Unit, Leicester General Hospital, Leicester

\section{REFERENCES}

1 Surawy C. Knowledge about diabetes in type 1 patients is related to metabolic control. Diabet Med 1989:6:784-6.

2 Tan AS, Yong LS, Wan S, et al. Patient education in the management of diabetes mellitus. Singapore Med J 1997;38:156-60.

3 Choy DK, Tong M, Ko F, et al. Evaluation of the efficacy of a hospital-based asthma education programme in patients of low socioeconomic status in Hong Kong. Clin Exp Allergy 1999;29:84-90.

4 Haines ST. Patient education: a tool in the outpatient management of deep vein thrombosis. Pharmacotherapy 1998;18:158-64.

5 Choi PM, Nugent FW, Schoetz DJ, et al. Colonoscopic surveillance reduces mortality from colorectal cancer in ulcerative colitis. Gastroenterology 1993;105:418-24.

6 Karlen P, Kornfeld D, Brostrom O, et al. Is colonoscopic surveillance reducing colorectal cancer mortality in ulcerative colitis? A population based case control study. Gut 1998;42:711-14

7 Lindberg B, Persson B, Veress B, et al. Twenty years' colonoscopic surveillance of patients with ulcerative colitis. Detection of dysplastic and malignant transformation. Scand J Gastroenterol 1996;31:1 195-204.

8 Provenzale D, Onken J. Surveillance issues in inflammatory bowel disease: ulcerative colitis. J Clin Gastroenterol 2001;32:99-105.

9 Eaden JA, Abrams K, Mayberry JF. The Crohn's and colitis knowledge score: a test for measuring patient knowledge in inflammatory bowel disease. Am J Gastroenterol 1999;12:3560-6. 
10 Eaden JA Abrams K, Ekbom A, et al. Colorectal cancer prevention in ulcerative colitis: a case-control study. Aliment Pharmacol Ther 2000; 14:145-53.

11 Altman DG. Practical statistics for medical research. 7th Ed. London: Chapman \& Hall, 1996.

12 Pinczowski D, Ekbom A, Baron J, et al. Risk factors for colorectal cancer in patients with ulcerative colitis: a case-control study. Gastroenterology 1994; 107: 117-20

13 Moody GA, Jayanthi V, Probert CSJ, et al. Long term therapy with sulphasalazine protects against colorectal cancer in ulcerative colitis: a retrospective study of colorectal cancer risk and compliance with treatment in Leicestershire. Eur J Gastroenterol Hepatol 1996;8: $1179-83$

14 Ransohoff DF. Colon cancer in ulcerative colitis. Gastroenterology 1988;94:1089-91

15 Ekbom A, Helmick C, Zack M, et al. Ulcerative colitis and colorectal cancer. A population-based study. N Engl J Med 1990;323:1228-33

16 Lennard-Jones JE, Young GP, Rozen P, et al, eds. Prevention and early detection of colorectal cancer. Prevention of cancer in inflammatory bowel disease. London: W B Saunders, 1996: 217-38.

\section{IMAGES IN MEDICINE}

\section{Wallenberg's lateral medullary syndrome}

\begin{abstract}
$A^{p}$
previously healthy 55 year old man presented with acute onset of nausea, vomiting, diplopia, and vertigo. He had hoarseness of voice, difficulty in swallowing, and nasal regurgitation of food and water. He had a tendency to fall to the right side. On neurological evaluation he had cerebellar type of dysarthria, right sided gaze evoked nystagmus, paralysis of palatal and pharyngeal movement, and absent gag reflex on the right side. He also had Horner's syndrome on the right side. There was no motor weakness, deep tendon reflexes were normal, and both plantars were flexor. There was loss of pain and temperature sensations over left half of face, arm, and leg and presence of cerebellar signs on the right side. The rest of the examination including fundus was unremarkable. A provisional diagnosis of right sided lateral medullary syndrome was made. Unusually in this case, the contralateral half of face was involved. This may be due to involvement of the contralateral trigeminothalamic tract that crosses the side of the lesion within the dorsal medulla. Alternatively medullary neurons may receive input from the contralateral face. Investigations revealed high fasting $(9.1 \mathrm{mmol} / \mathrm{l})$ and postprandial $(13.3$ $\mathrm{mmol} / \mathrm{l}$ ) plasma glucose concentrations, a glycated haemoglobin of $10.5 \%$, total cholesterol of 7.0 $\mathrm{mmol} / \mathrm{l}$, low density lipoprotein of $4.9 \mathrm{mmol} / \mathrm{l}$, and high density lipoprotein of $0.9 \mathrm{mmol} / \mathrm{l}$. Magnetic resonance imaging revealed a small infarct in right lateral medullary region (fig 1 ). The patient was managed with insulin, aspirin, and atorvastatin. His condition steadily improved over the next two weeks and he was nearly asymptomatic six months after discharge.
\end{abstract}

S K Jain, A K Gupta, N Agarwal Department of Medicine, Lady Hardinge Medical College, and Dr Ram Manohar Lohia Hospital New Delhi, India

Correspondence to: Dr Alok Kumar Gupta; alok_bgupta@yahoo.co.in

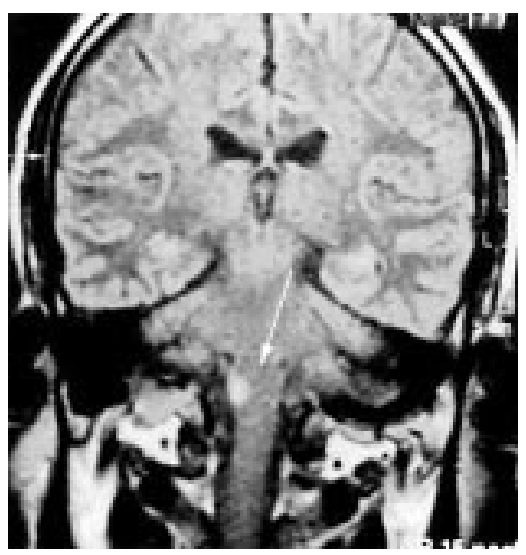

Figure 1 Magnetic resonance imaging scan showing tiny infarct in lateral medulla of right side. 